Mots. Les langages du politique

Les livres de journalistes politiques

\title{
Épistémologie du concept d'opinion publique en Corée et au Japon
}

Epistemology of the concept of public opinion in Korea and Japan

In-sook Choi

\section{OpenEdition}

\section{Journals}

Édition électronique

URL : https://journals.openedition.org/mots/21604

DOI : $10.4000 /$ mots. 21604

ISSN : 1960-6001

Éditeur

ENS Éditions

\section{Édition imprimée}

Date de publication : 19 mai 2014

Pagination : 77-94

ISBN : 978-2-84788-542-2

ISSN : 0243-6450

Référence électronique

In-sook Choi, «Épistémologie du concept d'opinion publique en Corée et au Japon », Mots. Les

langages du politique [En ligne], 104 | 2014, mis en ligne le 19 mai 2016, consulté le 24 avril 2022. URL:

http://journals.openedition.org/mots/21604; DOI : https://doi.org/10.4000/mots.21604 


\section{Épistémologie du concept d'opinion publique en Corée et au Japon}

En Extrême-Orient (극동아시아), deux pays qui partagent une terrible histoire, la Corée du Sud (한국) et le Japon (日本), se sont hissés en quelques décennies dans le groupe des pays économiquement développés. L'Organisation de coopération et de développement économiques (Classement en termes de PIB, 2011) place le Pays du Matin calme au vingt-cinquième rang, et le Pays du Soleil levant au troisième. L'un et l'autre se sont dotés, à l'issue de la seconde guerre mondiale, d'une constitution.

En 1919, le gouvernement temporaire de la république de Corée avait adopté une première constitution, mais elle resta lettre morte du fait de l'occupation japonaise. La Constitution coréenne (대한민국헌법, Daehanminkuk hunpup) de 1948, la première dans l'histoire à avoir entraîné l'instauration de nouvelles institutions, a été promulguée sous la présidence de Seung Man Lee. Elle a été modifiée neuf fois, le dernier changement, en 1987, introduisant l'élection du président de la République au suffrage universel direct et renforçant les pouvoirs de l'unique chambre, ce qui ouvrit la porte à la démocratisation, après des années de dictature militaire.

Au Japon, dès 1868, Mutsuhito (睦人), dont les pouvoirs venaient d'être rétablis, promit à son peuple une constitution. Celle-ci fut proclamée le 11 février 1889. Le texte se rapproche de la Constitution prussienne de 1850. L'Empereur, de droit divin, consent à associer la nation à sa souveraineté en déléguant certains pouvoirs à la Diète, qui se compose de deux Chambres, la Chambre des Pairs et celle des Représentants. En 1947, la Constitution de l'État du Japon (日本国憲法, Nihonkoku kenpô) a mis en place un parlement bicaméral. Le rôle tout à fait symbolique de l'Empereur permet de classer le pays dans la liste des démocraties.

La vox populi, c'est-à-dire l'opinion publique, est auscultée par les organismes de sondages, dans un parallèle avec le suffrage universel qui consacre le principe "un homme - une voix». Comment cette vox populi, dans ces deux anciennes royautés aux racines confucéennes, a-t-elle trouvé à s'exprimer au 
cours des siècles précédant l'instauration de systèmes politiques directement inspirés de modèles occidentaux?

Chaque année, plus d'un millier de sondages d'opinion sont réalisés et publiés dans ces deux pays asiatiques, tout comme en France ${ }^{1}$. Ils visent à recueillir l'expression de l'opinion publique sur différents sujets d'ordre politique, économique et social. Comprendre comment les résultats des sondages ont pu se substituer au concept d'opinion publique et en monopoliser le sens dans ces deux pays à tradition confucéenne n'est toutefois pas évident. Le sens de l'expression susceptible de traduire opinion publique, dans son dernier avatar, tend à faire oublier les significations précédentes qui ont fini par se confondre dans une acception moderne et communément admise.

Aujourd'hui, les résultats des sondages seraient considérés comme l'image, fidèle et non déformée, de l'opinion publique, bien que les sociologues des médias, Bourdieu (2002) notamment, nient la validité des sondages et affirment que «l'opinion publique n'existe pas». L'enquête d'opinion est un miroir qui reflète, cristallise et condense l'état de l'opinion, tout comme le sondage géologique qui remonte des profondeurs du sol une carotte, permet de mettre au jour ce qui était caché et d'en analyser les composantes ainsi que l'histoire.

En fait, il serait plus sensé de confronter ce qu'on peut considérer comme une étrangeté ou une originalité (c'est-à-dire la tendance à définir l'opinion par la pratique des sondages) à des figures plus anciennes (Blondiaux, 1998), c'est-à-dire à l'histoire des significations qui ont conduit à un contenu monolithique et consensuel du concept. C'est dans la dialectique entre passé et présent, le passé étant soumis à la censure du présent, que l’on peut apprécier la valeur profonde du concept d'opinion publique.

Dans cette perspective, on fera l'hypothèse que le développement du concept d'opinion publique en Corée et au Japon suit le même parcours que son homologue occidental dans le processus de naissance d'une opinion publique et d'évolution du sens donné au vocabulaire qui y réfère. Seuls changent les mots pour le dire, l'usage plus tardif des sondages et l'installation, elle aussi plus tardive, de la démocratie.

Cette recherche interroge donc des facteurs qui répondraient ou non d'une évolution similaire de l'opinion publique dans les systèmes sociopolitiques coréens et japonais par rapport aux sociétés occidentales.

Pour la méthodologie comparative, cette étude atteint ses limites en termes de données concernant la Corée alors qu'au Japon, nombreuses sont les études

1. En France, 850 enquêtes d'opinion ont été publiées en 2006, d'après Patrick Lehingue dans Subunda. Coups de sonde dans l'océan des sondages (2007); au Japon, 1218 sondages ont été réalisés en 2007, d'après Cabinet Office, Government of Japan. En Corée, ce genre de statistique n'existe pas. J'évalue à 400 millions de dollars US le chiffre d'affaires des instituts de sondage en 2009 . Ce montant est cinq fois plus élevé que celui de l'année 2000 , qui atteignait 87 millions de dollars. 
et les contributions sur le thème de l'opinion publique. D'où la difficulté d'une analyse comparative plus poussée. Le dessein général reste l'articulation des contextes historiques successifs desquels émerge une opinion publique, puis la technique des sondages.

Le concept d'opinion publique n'a pas vu le jour en Asie. Il est importé de l'Occident. C'est la raison pour laquelle les données disponibles dans ce domaine sont rares en Corée et au Japon. Ce n'est que depuis peu que quelques universitaires japonais publient des articles et des ouvrages sur l'évolution du concept d'opinion publique, ce genre d'étude n'étant pas encore développé en Corée. À défaut de données consistantes, notre étude s'appuie sur l'histoire du mot yôron ${ }^{2}$ et la fluidité de son sens en fonction de l'évolution des structures sociales.

\section{L’origine de l'opinion publique en Corée et au Japon}

\section{L'expression opinion publique}

En Occident, du point de vue étymologique, l'expression opinion publique est composée de deux notions : opinion et public. L'opinion publique est en effet d'abord celle des élites qui rendent «publiques» leurs opinions sur les affaires du royaume (Champagne, 1990). Il y a donc deux champs différents qui se marient dans le couple conceptuel opinion publique.

En revanche, en Corée et au Japon, cet ensemble est condensé en une seule notion, dont «public » est absent et qui est désignée par le mot yôron. En effet, le terme «public» (公衆: kôshû en japonais; 공중 : gongjung en coréen) existe dans les deux pays, mais il n'est normalement pas associé au concept d'«opinion ». Ce terme, présent depuis l'Antiquité, provient du chinois (公衆 : gongzhong). On l'emploie notamment dans les cas suivants : parler en public, hygiène publique (ici, «public » signifie « foule »), sens civique, comportement public. Pour désigner l'ensemble des citoyens, la société, le grand public, etc., le terme seken (世間) est utilisé au Japon, sesang saram (세상사람) en Corée. C'est pourquoi la notion de yôron repose sur une ambiguïté. Il est difficile de déterminer le moment précis où le yôron s'est imposé dans ces deux sociétés.

En Occident, à partir de la fin du Xvie siècle, la lexie opinion publique s'emploie pour désigner l'ensemble des idées et des jugements partagés par un groupe social ou une partie de ce dernier (Blondiaux, 1998). L'apparition du concept d'opinion publique à la fin de l'Ancien Régime a enrichi le vocabulaire politique (Habermas, 1992).

2. Dans le passé, la translittération yôron était utilisée en Corée. Elle devint yeoron il y a quinze ans. Nous adoptons cependant yôron car le mot japonais, lui, est romanisé en yoron, sans circonflexe. 
Cette conception de l'opinion publique semble apparaître au Japon et en Corée, respectivement, à l'ère Meiji (明治) et sous la dynastie Joseon (조선), à la fin du XIX $\mathrm{X}^{\mathrm{e}}$ siècle. Une interrogation demeure : pourquoi si tardivement? En premier lieu, l'organisation sociale traditionnelle en castes et en communautés dans ces deux pays confucéens était très rigide. Leur transformation et leur modernisation ont été très lentes. En second lieu, l'importation et l'adaptation des institutions démocratiques venues de l'Occident, telles que les partis politiques, la presse de masse et les mouvements sociaux, se sont effectuées plus tard.

En remontant dans le temps, on retrouve plusieurs mots qui ont signifié pendant de courtes périodes opinion publique avant que yôron en monopolise le sens, au terme d'une longue histoire. Ce sont (en coréen puis en japonais) :

- 공론 (Gongron); 公論 (Kôron),

- 공의 (Gong. ui) ; 公議 (Kôgi),

- 민의 (Min. ui); 民意 (Min. i),

- 인심 (Insim); 人心 (Jinshin),

- 중론 (Jungron); 衆論 (Shûron), etc.

\section{L'origine de yôron}

Quand commence-t-on à utiliser le mot yôron en Corée et au Japon ? D’où vientil ? C'est en Chine qu'il apparaît sous la forme 輿論 (yulun)3, dans Liangshu (梁 書), consacré à l'histoire politique de la dynastie Liang (梁朝, 502-557)4 (Satô, 2007). Ce terme signifie «renommée» (Nishihira, 2009) et réfère donc à un jugement de valeur porté sur une personne. Il renvoyait au mode de sélection des fonctionnaires sous le règne de l'empereur Wudi (武帝, 502-549)5. La question était alors de savoir s'il fallait s'en remettre aux élites en raison de leurs connaissances approfondies ou bien écouter le 輿論 (yulun). La Cour voulait recueillir l'avis des sujets sur la sélection de ses gouverneurs. Cette méthode rappelle le sultan Hārūn al-Rashīd des Mille et une nuits qui circule déguisé dans Bagdad pour connaître le sentiment du peuple à l'égard de sa façon de gouverner. Mais ce yulun ressemble plutôt à la fama popularis, aux rumores de Rome, qu'à une véritable opinion publique.

Le mot (輿論) est arrivé en Corée dans les bagages des lettrés et de la culture chinoise avant de passer dans l'archipel nippon. Coréens et Japonais lui donnèrent le même sens.

3. Le 輿 signifie «public» ou «masse», 論 «opinions». Cependant ces caractères ne sont pas utilisés séparément.

4. La dynastie Liang (Chine du Sud) fut précédée par la dynastie Qi du Sud et suivie par la dynastie Chen.

5. Le règne de Wudi fut des plus prospères et des plus stables. Il fit des efforts pour réconcilier les intellectuels et le peuple (http://www.angelfire.com/co/leong/history7.html). 


\section{Le développement du yôron en Corée}

\section{Avant l'apparition de groupes sociaux structurés}

En Corée, plusieurs mots déjà mentionnés faisant référence à l'opinion publique apparaissent dans l'Histoire de la dynastie Goryeo (고려사), ouvrage publié en $1451^{6}$. Il importe de ne jamais perdre de vue la distinction que nous effectuons entre l'opinion attachée à une période historique précise qui a une signification circonstanciée, et l'opinion publique dans notre conception moderne issue des sondages.

Deux mots, gongron (공론)7 et yôron (여론), qui signifient «opinion publique », revêtent une grande importance pour notre étude. Le premier désigne l'opinion des élites (grec : logos), c'est-à-dire de la classe aristocratique Yangban, considérée comme impartiale, solide, juste, car issue des discussions et des réflexions des élites savantes et s'imposant dès lors au peuple. Gongron $^{8}$ serait aussi dérivé du chinois. Ce mot est mentionné dans Xu Zizhi Tongjian 續資治通鑑 [Histoire chinoise des dynasties Song et Yuan] de Bi Yuan (畢沅, 1730-1797)9, avec une cinquantaine d'occurrences (Lee, 2002).

Yôron, l'opinion du peuple (grec: doxa), ou la manifestation du ressenti et du sentiment communs (Kim, 2002) est l'expression des émotions et des sentiments de personnes qui ne réfléchissent pas rationnellement, qui ne sont pas objectives et sont, à ce titre, partiales et injustes (Lee, 2002). On emploie simultanément yôron et minsim (민심: «voix du peuple»).

Dès le $x v I^{e}$ siècle, deux philosophes coréens insistèrent sur la nécessité, pour les politiques, de prendre en compte les aspirations de l'opinion publique. Cho Kwang-Cho (조광조 : 1482-1519) et Yi I (이이 : 1536-1584) firent l'effort d'instaurer une relation entre la classe éclairée et le peuple.

Cho estimait que seul le gongron était à prendre en considération. II recommandait aux élites politiques d'être à l'écoute des attentes, des suggestions, des critiques qu'il véhiculait, ce qui permettait d'assurer une relation harmonieuse entre la classe dirigeante et le peuple et d'instaurer une relation de confiance entre ces deux mondes.

Yi pensait qu'aux lettrés (사림 : sarim), forts de leurs connaissances,

6. Le spécialiste taïwanais de l'opinion publique, Zhu Chuan Xing, considère que le mot yulun fut remplacé par minyi (民意) auxıxe siècle (Kim, 2002). Aujourd'hui, les Chinois utilisent les deux termes, Min. yi et Yulun, pour désigner l'opinion publique.

7. D'après Naver English Dictionary, Gongron a sens suivants: 1. Public [popular, general] opinion, 2. Synonyme du yôron [http://endic.naver.com/krenEntry.nhn?sLn=en\&entryld=802fob5892 9c424b97abfooc3bc871a3\&query=\%EA\%B3\%B5\%EB\%A1\%Ao/2014/01/o6].

8. Le confucéen chinois Zhu Xi (朱子, 1130-1200) a conceptualisé gongron 公論 en lui donnant les significations suivantes : «la parole du peuple (萬ロー辭) », « des opinions bien arrêtées ».

9. Il était collectionneur, éditeur et historien à l'époque Qing 清 (1644-1911). 
incombait la responsabilité de former et d’éduquer le peuple et de gérer les affaires publiques avec compétence et modestie, selon les préceptes confucianistes, en consultant toutes les composantes de la société, y compris les plus modestes et les moins considérées $\left(Y i\right.$ I, 1972) ${ }^{10}$.

La recherche d'un consensus en serait, du même coup, facilitée, ce qui est la condition nécessaire à un fonctionnement sans heurt de la vie sociale (Kim et al., 1994). Le primat était donné au yôron, aux dépens du gongron. La multiplication et la diversification des canaux de communication par lesquels le yôron s'exprime étaient souhaitées (Jeon, 2007).

Cette conception de l'opinion publique, distribuée en deux volets selon qu'elle émerge de l'élite ou de la masse populaire, ne s'inscrivait pas dans l'apparition d'une nouvelle culture politique venue de l'Occident. Une élite exerçant une critique permanente vis-à-vis du pouvoir politique par l'information, la discussion et l'usage du raisonnement ne pouvait exister dans la société coréenne du xvı e siècle. Jusqu'à la Révolution Donghak (동학, savoir de l'Orient) ${ }^{11}$ de 1894, celle-ci était hiérarchisée, traditionnelle, répartie en castes. Le climat sociopolitique et économique était différent de celui de l'Occident. Cette recherche montre les timides signes d'une prise en considération de l'opinion du peuple par le pouvoir royal.

\section{Élargissement du yôron}

Le yôron (여론), opinion du peuple, a commencé à se manifester dans son interprétation moderne aux alentours de 1890, époque de changements sociaux et d'émergence d'une presse de masse.

La Révolution Donghak en 1894 et ses insurrections paysannes représentèrent un moment décisif dans la lutte contre le système féodal et une société hiérarchisée. Elles se réclamaient du minjung (민중 : groupe nationaliste) et s'appuyaient sur les basses classes de la société (paysans, commerçants, artisans). Le mouvement Donghakest d'autant plus significatif, pour un chercheur qui s'intéresse à l'histoire sociale de l'opinion publique, qu'il était aussi soutenu et organisé par une nouvelle classe, la petite bourgeoisie, désireuse d'assurer le développement du pays et l'autonomie de celui-ci vis-à-vis de la Chine et du Japon.

Cette révolution visait à créer un monde où l'égalité régnerait en éradiquant les systèmes féodaux. Ont été abolis le système des classes et le gwagô (과거), ce mode de recrutement des fonctionnaires qui privilégiait la connaissance des textes confucianistes et réservait de facto aux enfants des familles nobles l'accès aux postes importants. En ce sens, le mouvement Donghak représente le début de la modernisation de la société et de la pensée coréennes. D’ailleurs,

10. Yulkok chonsuh, vol. III, al. 33 .

11. Voir infra. 
les concepts occidentaux de "public » et de "privé » ont été introduits sous l'influence du Japon. Une réforme très importante fut adoptée : la séparation de la «cassette royale » et du Trésor public. Au-delà de l'aspect comptable, cette réforme a une portée hautement symbolique.

Selon la reconstitution désormais classique proposée par Habermas, cette mutation idéologique a été rendue possible par - et s'est appuyée sur - une transformation des systèmes de relations économiques et de la sphère familiale bourgeoise, la naissance d'une bourgeoise éclairée et la création de lieux et d'institutions (clubs, cafés littéraires, presse...) (Habermas, 1992).

Le Conseil de l'Indépendance (독립협회), club créé en 1896 par des penseurs progressistes, élargit son recrutement. À la fin de la même année, 2000 personnes y avaient adhéré, telles que des commerçants, des étudiants, une nouvelle classe intellectuelle. Transformé en association, il lança le journal The Independent (독립신문 : Dongnip Sinmun, en hangul) qui paraissait trois fois par semaine. Les médias modernes étaient ainsi durablement introduits dans la société coréenne (Kim, 2006).

La ligne éditoriale du Dongnip resta neutre, jusqu'à son interdiction en 1900. Pendant quatre ans, le journal fut un canal de circulation d'informations entre le gouvernement et les classes populaires, qui ne se privaient pas de critiquer les mesures politiques et de mettre en lumière les « affaires » et les dossiers mettant en cause les hommes politiques corrompus ou corrupteurs ( $\mathrm{ibid}$.).

De nouveaux organes de presse s'engagèrent dans la même voie : Maeil (매일), Hwangsung (황성) et Chekook (제국) virent le jour en 1898. De la sorte, le yôron vit son influence s'accroître, au sens où Tarde l'entend, en créant une communauté de lecteurs partageant des idées communes et des aspirations similaires vis-à-vis des certains idéaux politiques.

Le journal, accoucheur de l'opinion publique? Oui, si l'on en croit l'éditorial du Dongnip (22 août 1896) : «À la lecture de nos pages, les citoyens peuvent penser à ce qu'ils n'ont jamais pensé, et connaître ce qu'ils ignorent. De la sorte, ils peuvent se forger une opinion solide, car assise sur des réalités. Éducatrice majeure, la presse contribue à l'émergence d'une opinion. $\gg^{12}$

D'où venaient ces idées nouvelles? Le directeur de Dongnip Sinmun, Suh Chae-P'il (서재필, 1864-1951), diplômé de l'université George Washington, était fasciné par le New Journalism de Joseph Pulitzer et puisait son inspiration dans les colonnes du St. Louis Post-Dispatch, dont celui-ci était le rédacteur en chef (Lee, 2003).

Son journal était édité en anglais et en coréen, et pour cette version-ci utilisait exclusivement l'alphabet local, le hangul'13. La lecture et la compréhension

12. Éditorial du journal Dongnip, $1^{\mathrm{er}}$ mai 1896.

13. Dès lors, les Coréens utilisent le mot yôron en caractères chinois et coréen, respectivement 輿 論 et 여론. 
par les classes moyennes, qui ne savaient pas lire les caractères chinois, s'en trouvaient facilitées. Suh s'efforçait de mobiliser ses lecteurs, de leur faire prendre conscience de l'urgence de la lutte contre les impérialismes chinois et japonais. Mais son lectorat était très limité en raison du prix élevé de l'abonnement. Il contribua néanmoins à l'éducation politique des Coréens et à la promotion des idées invitant à une ouverture sur l'extérieur. Suh abordait des sujets ayant trait à l'actualité occidentale tels que les droits de l'homme, les libertés individuelles...

Soulignons le rôle de l'association Hyupsunghoe (협성회), dont les deux cents membres organisèrent une cinquantaine de débats publics afin de faire prendre conscience aux participants du poids qui pouvait être le leur et de l'influence qu'ils pouvaient exercer sur les orientations du gouvernement.

\section{Contestation grandissante de la colonisation japonaise}

L'émergence du yôron (여론) en Corée est aussi fortement liée à la lutte contre l'impérialisme nippon. À partir de 1910, date de l'annexion de la Corée par le Japon, la nature de l'opinion publique changea, ou du moins prit une nouvelle forme. Une critique contre l'oppression japonaise surgit alors des profondeurs du peuple coréen, et celui-ci se mobilisa de plus en plus, dans tout le pays, d'une manière violente. La réaction populaire atteignit son apogée lors de la grande manifestation du 1er mars $1919^{14}$, où les Coréens, sous le slogan «Vive l'indépendance », défilèrent dans les rues de Séoul. Ce moment décisif correspond à une prise de conscience, à l'échelle nationale, de la nécessité de lutter contre la domination japonaise et de résister à l'ennemi. Les mouvements syndicaux ont manifesté à leur tour, dans les années vingt, contre les entreprises japonaises qui exploitaient les ouvriers coréens.

Cet événement suscita l'extériorisation d'une opinion proprement dite, un ensemble de jugements et de sentiments partagés, une volonté générale de se révolter contre la colonisation, un désir commun de liberté (Tarde, 1989). Le même phénomène se reproduisit le 15 août 1945 lors de la libération de la Corée, consécutive à la capitulation du Japon.

La promotion des valeurs démocratiques et l'usage des enquêtes d'opinion par les sciences sociales ont participé à la légitimation des sondages en général en les parant d'une noble cause : ils offriraient aux gouvernés un moyen simple, direct et permanent d'expression publique, une sorte de contrôle des gouvernants qui pallierait le sentiment d'exclusion dans les affaires publiques. Ainsi, au Japon comme en Corée, les sondages n'apparaissent qu'avec la démocratisation amorcée sous le «protectorat» américain après 1945 (Choi, 2008).

14. Le $1^{\text {er }}$ mars 1919 , les obsèques du dernier roi Kojong avaient attiré des foules venant de toutes les régions du pays. 


\section{Naissance de l'opinion publique au Japon}

\section{Apparition du yoron}

Il n'est pas évident de situer historiquement le moment précis où les Japonais ont commencé à utiliser le terme correspondant peu ou prou à opinion publique. Deux mots sont attestés dans les archives : yoron (輿論) et seron (世 論). Le premier désigne l'opinion des élites, c'est-à-dire de la famille impériale, des militaires, du clergé; le second, celle des classes populaires.

Le terme yoron (輿論) figure dans le livre Recueillir l'opinion des élites (奥 論の帰する所 Yoron no kisuru tokoro) paru vers 1415. On le trouve également dans Les récits de Surugadai (駿臺雜話 Surugadai zatsuwa) de Murô Kyûsô (室鳩巣 : 1658-1734). Le yoron est utilisé à nouveau par le dernier shogun, Tokugawa Yoshinobu (徳川慶喜), en 1867, alors qu'il cherche à faire évoluer le monde politique (Yamamoto, 1992). Apparemment, Yoshinobu voulait réunir les élites savantes autour du régime shogunal pour redresser le pays qui avait été mis à mal, le yoron devenant une sorte de machine idéologique.

Avec seron (世論), nous avons affaire en revanche à un néologisme de l'ère Meiji pour désigner l'opinion publique. Le mot apparaît dans l'ouvrage de Fukuzawa Yukichi, Abrégé d'un traité de civilisation (文明論之概略) de 1875, et signifie «l'opinion des gens du commun» (Miyatake, 2007). Le modèle politique issu de la Restauration Meiji avait généré une situation sociale favorable à une réelle prise de conscience de la valeur de l'opinion publique.

Celle-ci se confirmera avec l'émergence de la presse de masse. Les journaux ont accompagné ce phénomène de «cristallisation » d'une opinion publique, notamment les organes qui affichaient une opposition politique dans leurs éditoriaux (Séguy, 1983). Le premier véritable quotidien japonais, le Yokohama Mainichi Shimbun (横浜毎日新聞), apparut en 1871. Un an après, cinq journaux paraissaient régulièrement : le plus important d'entre eux, le Zasshi (雑 誌 magazine), était patronné par Kido Takayoshi15 (木戸孝允:1833-1877) (Huffman, 1980). Le moins virulent paraît avoir été le Tôkyô Nichinichi Shimbun (東 京日日新聞) de Fukuchi Gen. ichirô (福地源-郎), qui brocardait aussi le gouvernement, mais sans prendre parti systématiquement pour l'Assemblée (Esmein, 1991). Certains journaux demeuraient neutres, se bornant à se faire l'écho du sentiment populaire ou de l'opinion dominante des citoyens.

La dissolution des structures rigides de la société féodale et l'ouverture à l'Occident, accompagnées par l'introduction des connaissances modernes, ont favorisé ces nouvelles formes d'expression journalistique destinées à des publics encore très étroits (Esmein, 1991).

15. Homme politique pendant la fin du shogunat Tokugawa et l'ère Meiji. 
Aux alentours de 1890, les journaux japonais entrèrent dans une ère commerciale (Smith, traduit par Choi, 2005). Ils tiraient déjà à des dizaines de milliers d'exemplaires (Huffman, p. 386) et véhiculaient une culture de masse (Pons, 1988). La réalité de la force de l'opinion s'affirmait déjà à tel point que le gouvernement cédait à sa pression. On peut dire alors que le concept désigne, comme en son sens primitif, une instance de contrôle qui s'impose à l'action de chacun comme à celle des gouvernants (Blondiaux, 1998).

En ce sens, les journalistes, hommes politiques et penseurs, notamment Fukuchi Gen. ichirô et Fukuzawa Yukichi, mobilisaient l'opinion publique en utilisant particulièrement les mots yoron (輿論) et seron ou seiron (世論) ${ }^{16}$ dans leurs journaux, le Tôkyô Nichinichi Shimbun (東京日日新聞) et le Jiji Shinpô (時事新 報). Seron/seiron signifie «climat social», alors que yoron veut dire « opinion raisonnable». Le Dictionnaire anglo-japonais de Francis Brinkley (1896) donne les définitions suivantes: $y o r o n=~ « p u b l i c$ opinion », seron = «popular sentiment».

Les hommes politiques de l'ère Meiji distinguaient nettement les deux termes. Analysant le journal de Hara Takashi (Hara Takashi nikki 原敬日記) ${ }^{17}$, Sumitomo (2001) donne à yoron le sens d'une discussion débouchant sur un accord et à seron celui d'un débat qui s'élève entre deux partenaires aux points de vue discordants.

\section{Deuxpionniers}

Deux intellectuels ont particulièrement marqué l'évolution de l'opinion publique au Japon.

Fukuchi Gen. ichirô (福地源一郎 : 1841-1906), sujet du shogun à l'époque d’Edo, devenu journaliste du Tôkyô Nichinichi Shimbun (東京日日新聞) à l'ère Meiji, était un bon connaisseur de la politique intérieure et internationale. Il a importé des éléments de la culture occidentale, dont le mot society introduit pour la première fois dans l'archipel nippon. Il portait un vif intérêt au yoron (輿論) et au seron (世論), mentionnant quatre fois le premier et quatorze fois le second dans son livre (2003 [1898]) intitulé Hommes politiques de la fin $d^{\prime} E d o$ (幕末政治家). On y lit par exemple le passage suivant : «[...] pour calmer l'emportement du gouvernement et du yoron.» Dans ce contexte, yoron signifiait «l'opinion rationnelle», «l'opinion des gens de lettres». Pour le seron, il écrit : «le seron de l'époque et le comportement d'Andô ${ }^{18}$ ont été incriminés» (2003 [1900], p. 192), c'est-à-dire «les gens», «le monde» ou «la rumeur».

16. Notons que le mot 世論 pouvait se lire aussi bien «seiron» que «seron » à l'ère Meiji. Mais la première lecture a disparu au fil du temps.

17. Documents historiques relatifs aux ères Meiji et Taishô.

18. Andô Nobumasa (安藤信正 : 1819-1871) était un militaire qui eut une grande influence sur la politique à la fin d’Edo. Il voulut négocier un mariage d'intérêt entre le shogun Tokugawa Yoshitomi (徳 川慶福) et la princesse Kazunomiya (和宮) afin de réhabiliter l'autorité du régime shogunal, mais la princesse était déjà fiancée au prince Arisugawa Nomiyataruhito (有栖川宮 熾仁) : Shibahara, 1975, p.136-137. 
Fukuzawa Yukichi (福沢諭吉 :1834-1901), fondateur du journal Jijishinpô (時 事新報), connaissait également l'Occident pour avoir beaucoup voyagé comme envoyé du gouvernement japonais aux États-Unis et en Europe. Il introduisit la culture et les idées occidentales au Japon. Partisan de l'égalité homme/femme, il mit en cause la morale confucéenne qui encourageait l'homme à jouir de sa supériorité sur la femme et légitimait la polygamie ${ }^{19}$.

Prenant en compte l'importance de l'égalité, il insista sur le seron (世論) dans son ouvrage Abrégé d'un traité de civilisation. Il y avance qu'on peut connaître le vrai paysage de la société japonaise à partir du seron, expression de la volonté des gens du commun, et défend ce point de vue afin de favoriser l'ouverture du Japon à la civilisation occidentale.

\section{Influence croissante du seron}

Comme en Occident, le Mouvement pour la liberté et les droits du peuple (le Jiyû minken undô 自由民権運動 : 1874-1890) au Japon joua un rôle majeur dans l'évolution de l'opinion publique. Les élites intellectuelles, la bourgeoisie urbaine et les classes populaires se mobilisèrent contre les principes des gouvernements de l'ère Meiji afin de légitimer leurs revendications sociales et politiques. Elles réclamaient la mise en place d'un parlement et d'une constitution, ainsi que la liberté de la presse et le droit de réunion. L'opinion publique japonaise est active, et même bruyante, dès le lancement des premiers journaux. Les premières années du Mouvement pour la liberté et les droits du peuple en sont une illustration, ainsi que les très importantes manifestations, à Tokyo, pendant la crise diplomatique de 1889. Ce climat social porte en germe une structuration du seron.

Lors du conflit avec la Russie, en 1904, celui-ci devint une réalité. Dès le 10 juin 1903, sept professeurs de l'Université impériale de Tôkyô (東京帝国 大学) avaient proposé au Premier ministre Katsura Tarô (桂太郎) d'utiliser la presse populaire, les magazines et les réunions pour rallier le seron aux vues du gouvernement et s'en faire un allié. Les sociologues japonais Satô et Miyatake (2003) expliquent que le yoron (輿論) fut seronisé (世論化) de façon remarquable dans cette opération.

L'épanouissement de l'ère Taishô (大正 : 1912-1926) se trouva favorisé par un phénomène remarquable : la scolarisation massive de la population. En

19. Fukuzawa Yukichi est un penseur influent qui visait à éclairer l'esprit des Japonais. Il vulgarisa les idées démocratiques, notamment celle d'égalité : voir «L'Appel à l'étude - livre premier» (Gakumon no susume - shohen), dans Cent ans de pensée au Japon, t. Il (trad. C. Galan, Y.-M. Allioux éd., Arles, Philippe Picquier, 1996, p.7-20). Fukuzawa a laissé un adage très connu: "Le Ciel ne crée aucun homme supérieur ni aucun homme inférieur aux autres hommes» (Galan, op. cit., p. 11). Paradoxalement, son point de vue sur le colonialisme en Asie était en opposition avec les droits de l'homme. C'est pourquoi des controverses ont surgi quant à son égalitarisme, notamment en Corée, en Mandchourie et à Taïwan, qui furent colonisés par le Japon. 
effet, la généralisation de l’éducation obligatoire (義務教育) commence à faire sentir ses effets lorsque les générations concernées par la loi sur l'éducation de 1872 arrivent à l'âge adulte, au début des années 1890 (Galan, 2001). Un autre événement va contribuer à l'élargissement du seron : l'adoption du suffrage universel (普通選挙), réservé aux hommes, en 1925. Les élites politiques durent alors reconnaître le statut de citoyen à part entière à une partie de plus en plus importante de la population.

Dans ce climat, la ligne de démarcation entre le yoron (輿論) et le seron ( 世論) devint de plus en plus difficile à tracer (Satô, 2007). Les deux termes étaient considérés comme synonymes jusqu'à la deuxième guerre mondiale.

\section{L'ambiguïté du concept d'opinion publique}

À partir de 1946, le SCAP (Supreme Commander for the Allied Powers : 連合軍最 高司令官) a recueilli des données sur divers aspects relatifs à la société japonaise grâce à des enquêtes sociologiques et à des sondages d'opinion. Il disposait dans l'administration nipponne d'une section appelée PO \& SR (Public Opinion and Sociological Research) rattachée au département de la CIE (Civil Information and Education).

Une question de terminologie apparut. Dès novembre 1946, dans un souci de simplification de la langue écrite, le ministère de l’Éducation (文部 省) imposa une liste de 1850 kanji2o dits «usuels» (tôyô kanji : 当用漢字) en recommandant aux journaux, magazines, rédacteurs de documents officiels... d'éviter autant que possible les sinogrammes rares ou difficiles. Le kanji 輿 [yo] correspondant à la première syllabe ne faisant plus partie de cette liste, le composé 舆論 (yoron) ne fut dès lors plus utilisé, et l'usage alla à 世論 (seron) qui engloba le sens de yoron 輿論.

Désormais, la même graphie 世論 se prononce de deux façons : seron et yoron. En effet, la plupart des kanji ont plusieurs lectures possibles, et le kanji 世 peut se lire aussi bien « sé » que «yo ». D’après l'enquête réalisée par la NHK (Nippon Broadcast) en 1989 sur les habitudes linguistiques des habitants de Tôkyô âgés de plus de 16 ans, il apparaît que 63\% choisissent la prononciation yoron, $34 \%$ seron. Les Japonais ne sont pas contraints d'opter pour une prononciation en particulier. Le sociologue Satô (2003) ironise sur cette situation dans un article intitulé « Un Yoron japonais ambigu ». Il avance que le sens est différent selon la prononciation choisie pour la graphie 世論. De son côté, le statisticien Nishihira (2009) affirme qu'il n'est pas évident de faire la différence conceptuelle entre yoron et seron.

Nous avons constaté pour notre part que la plupart des sondeurs, journa-

20. Littéralement : «lettre chinoise» (idéogramme ou pictogramme). 
listes et universitaires prononcent yoron ${ }^{21}$, tout comme les adhérents de la JAPOR (Japan Association for Public Opinion Research : 日本世論調査協会) et les médias. C'est la raison pour laquelle nous romanisons le mot 世論 en yoron dans cette étude.

Après la naissance des sondages d'opinion, les politologues, les sociologues, les sondeurs et les journalistes japonais mirent en question la définition du yoron (世論). Pour le politologue Matsumoto (2003), il s'est agi d'abord de l'opinion partagée au sein d'un groupe. Mais celui-ci a finalement rejoint l'opinion du statisticien Hayashi, qui définit l' «opinion publique» (yoron) comme étant ce que mesurent les sondages (1976). Le journal Asahi donne de son côté cette définition (1976) : «attitude ou opinion de la majorité sur tel ou tel problème ». Pour le Yomiuri (1982), c'est la volonté générale du peuple sur telle ou telle affaire. Cependant, on voit que les deux dernières conceptions de l'opinion publique ne sont en rien nouvelles.

En résumé, les résultats des sondages d'opinion refléteraient l'état de «l'opinion publique» au Japon comme dans les autres pays démocratiques. Il semble bien, en effet, que les sondages d'opinion au travers de leurs utilisations quotidiennes, par la presse et les acteurs politiques, aient réussi leur «OPA» sur la notion d'opinion publique. Tout conduit à accepter désormais l'équivalence entre ce que mesurent les sondages et ce qu'il faut entendre par opinion publique (Blondiaux, 1997). Mais notons que la réflexion autour de l'opinion publique laisse toujours apercevoir un paradoxe étrange et souvent noté : il existe un contraste saisissant entre la fréquence des usages scientifiques et politiques de cette notion et les difficultés qui président à sa définition, entre sa longévité et sa labilité, sa résistance et son évanescence. Le désarroi des glossateurs se manifeste de manière souvent explicite. Parmi d'autres exemples, il est d'usage de rappeler que vers le milieu des années soixante, un manuel américain pouvait recenser plus d'une cinquantaine de définitions de la notion, partiellement irréductibles les unes aux autres (Childs, 1965).

Dans tous les cas, l'évolution entre le moment où s'exerce le monopole de l'opinion exprimée par les élites et celui où est prise en compte la manifestation d'une opinion reflétant celle de la communauté nationale peut être expliquée par l'histoire de l'organisation des sociétés japonaise et coréenne. L'opinion dont le peuple est porteur consiste souvent en des manifestations de désapprobation violentes visant à faire fléchir des gouvernants auxquels il est demandé de satisfaire des exigences relatives à certains aspects de l'existence. Ces mouvements sociaux représentent une image de l'opinion noire (Blondiaux, 1998).

21. Lors de la conférence du 28 novembre 2003 de la JAPOR sur les échantillonnages et les sondages électoraux, les conférenciers dont moi-même avons utilisé yoron pour désigner l'opinion publique. D'ailleurs, la revue trimestrielle de la JAPOR s'intitule よろん (Yoron). On ne trouve que yoron et yoron chôsa dans Le dictionnaire contemporain de la sociologie édité par Kôbundô [Koubundou] (弘文堂, 2012). 
Le tableau suivant récapitule les termes synonymes d'opinion suivant les personnes qui les incarnent.

\begin{tabular}{llll}
\hline & Chine $^{\star}$ & Corée & Japon \\
\hline Opinion des élites & gonglun & gongron & yoron \\
\hline Opinion du peuple & yu. lun, min. yi & yôron & seron \\
\hline $\begin{array}{l}\text { Terme actuel dési- } \\
\text { gnant l'opinion } \\
\text { publique }\end{array}$ & yu. lun, min. yi & yôron, min. ui & $\begin{array}{l}\text { yoron, seron, } \\
\text { min. i }\end{array}$ \\
\hline
\end{tabular}

Les termes chinois gonglun, yu. lun, min. yi sont aussi utilisés pour désigner l'opinion publique en Corée et au Japon. Mais seron n'existe ni en chinois ni en coréen. Nous supposons que l'usage du chinois a été similaire dans les deux pays, Chine et Corée. En arrivant au Japon via la Corée, le chinois s'est trouvé plus fortement transformé. Autrement dit, la Corée a été le premier pays à recevoir la langue chinoise, qu'elle a ensuite introduite au Japon.

*Dictionnaire chinois-français (1990).

Cette étude a tenté d'analyser l'évolution du concept d'opinion publique en Corée du Sud et au Japon. Cette notion est l'héritière de la longue histoire des relations entre ces deux pays, empreints de culture confucéenne, et dont le premier fut colonisé pendant trente-cinq ans par le second. Le processus de la naissance d'une opinion publique et de l'évolution du sens donné à ce mot présente une similitude avec ce qui se passa en Occident, en dépit des différences socioculturelles.

La liberté d'expression ne fut-elle véritablement consacrée dans l'Archipel nippon qu'après la deuxième guerre mondiale, le pays étant sous le contrôle des Alliés? Contredisant cette idée communément répandue, les historiens du Japon contemporain, dont Michel Vié (1995), la considèrent comme un cliché. Le contrôle des revues et journaux est introduit pour la première fois par l'ordonnance de presse de 1873; avec la suppression des dernières mesures de censure, en 1897, Fraser (1995) considère que la presse est dès lors libre. En Corée du Sud, ce sera en 1987, lors de la première élection présidentielle au suffrage universel direct.

Aujourd'hui, les lieux de production d'un discours sur l'opinion publique se diversifient et se structurent avec l'aide des nouvelles technologies de l'information et de la communication. On construit les réseaux sociaux de communication à l'échelle mondiale par le biais d'Internet, Facebook, Twitter, Cyworld pour la Corée, Mixi et GREE pour le Japon. En 2008, 75,2\% des Japonais et 75,8\% des Coréens accédaient à Internet 22 . Tarde avait déjà introduit en 1901 l'idée de la suggestion à distance. C'est « la pensée du regard d'autrui » par

22. World Data Bank, 17 juillet 2010. 
un phénomène de «contagion à distance » qui influence la formation de l'opinion chez des individus membres d'une communauté et isolés physiquement les uns des autres.

Bien que les études sur le concept d'opinion publique en Corée du Sud et au Japon soient très limitées, cela n'empêche pas l'usage de sondages visant à analyser les composantes de la société. Depuis les années quatre-vingt-dix, après l'effondrement des régimes communistes et l'émergence de la mondialisation, l'analyse comparative s'est développée. Bon nombre d'associations académiques ont été créées. Les travaux empiriques sur l'opinion se sont développés dans les sciences sociales à partir de grandes enquêtes internationales telles que World Value Survey, East Asian Barometers, Asian Barometers, etc.

S'agissant de ceux-ci, c'est le ministre de la Culture taïwanais qui prit l'initiative, en 2000, de réunir treize pays, dont la Corée du Sud et le Japon, afin de lancer des enquêtes assorties d'instruments de mesure et permettant de mesurer l'évolution de la pression démocratique et des systèmes de valeurs dans les pays adhérents. La crise mondiale ayant entraîné des réductions budgétaires, l'activité de ces «baromètres» s'en trouve affectée.

\section{Références}

Allioux Yves-Marie, 1996, Cent ans de pensée au Japon, t. II, Arles, Picquier.

BLoNDIAUX Loïc, 1998, La fabrique de l'opinion. Une histoire sociale des sondages, Paris, Le Seuil.

- 1997, «Ce que les sondages font à l'opinion publique», Politix, vol.10, n³7, p.117-136.

BoURDIEU Pierre, 2002 [1984], «L'opinion publique n'existe pas», Questions de sociologie, Paris, Minuit, p. 222-235.

Cham Pagne Patrick, 1994, Faire l'opinion. Le nouveau jeu politique, Paris, Minuit.

CHILDS Harwood, 1965, Public opinion. Nature, Formation and Role, Princeton, Van Nostrand.

CHOI In-sook, 2008, "Institutionnalisation des sondages d'opinion au Japon et en Corée du Sud », thèse, Sciences Po Paris.

ESMEIN Jean, 1991, Le Japon contemporain, Paris, PUF.

FRASER Andrew, 1995, Japan's early Parliaments 1890-1905, Londres, Routledge.

GALAN Christian, 2001, L'enseignement de la lecture au Japon, Toulouse, Presses universitaires du Mirail.

HABERMAS Jürgen, 1992 [1962], L'espace public, Paris, Payot.

Huffman James L., 1980, Politics of the Meiji Press: The Life of Fukuchi Gen. ichirô, Honolulu, The University Press of Hawaii.

Lehingue Patrick, 2007, Subunda. Coups de sonde dans l'océan des sondages, Bellecombe-en-Bauges, Éditions du Croquant.

NISHIHIRA Shigeki [NISIHIRA Sigeki] (1983), «Political opinion polling in Japan », Political Opinion Polling, New York, St. Martin's Press. 
Pons Philippe, 1988, Japon, Paris, Le Seuil.

SÉGuY Christiane, 1983, Histoire de la presse japonaise, Aurillac, Publications orientalistes de France.

TARDE Gabriel, 1989, L'opinion et la foule, Paris, PUF.

VIE Michel, 1995 [1971], Le Japon contemporain, Paris, PUF.

CHOI Jung-ho 최정호, 1982, Anron Munhwa-wa Daejung Munhwa 언론문화와 대중문화 [Cultures de communication et de masse], Séoul, Minumsa 민음사.

FUKUCHI Gen. ichirô 福地源一郎, 2003 [1900], Bakumatsu seijika 幕末政治家 [Hommes politiques de la fin d'Edo], Tôkyô, Iwanami shoten 岩波書店. 国民の友社、総日本 史籍協会叢書、1900 年発行、1979年復刻.

FuKUZAWA Yukichi 福沢諭吉, 1962 [1898], Bunmeiron no gairyaku 文明論之概略 [Abrégé d'un traité de civilisation], Tôkyô, Iwanami shoten 岩波書店.

— 1978 [1898], Gakumon no susume 学問のす \め [Invitation à l'instruction], Tôkyô, Iwanami shoten 岩波書店.

HaN Won-yong 한원영, 2002, Hankook Sinmun Hanseki 한국 신문 한 세기 [Un siècle de presse coréenne], Séoul, Purunsasangsa 푸른사상사.

KıM Se-chul et KıM Yong-je 김세철 · 김영재, 2000, Joseonsidae-ui Anron Munhwa 조선 시대의 언론문화 [La culture de communication de l'époque de Joseon], Séoul, Communication Books 커뮤니케이션북스.

KıM Young-ju 김영주, 2002, «Yijo Chogi Yôron Hyungsung Gwajung-é Daehan Yeonku» 이조초기 여론형성과정에 대한 연구 [«Recherche sur le processus de formation de l'opinion publique au début de la dynastie Yi en Corée»], Recherche sur la science linguistique 언어학연구, vol. II, n³ 3 , décembre.

KıM Young-jik 김용직, 2006, « Kehwaki Hankuk-ui Kundae-jeok Gongronjang-gwa Gongron Hyungsung Yeonku » 개화기 한국의 근대적 공론장과 공론형성 연구 : 독립협 회와「독립신문」을 중심으로 [«Étude sur l'espace public moderne et la formation de l'opinion publique en Corée. Le Conseil de l'Indépendance et le journal Dongnip »], Hankook Dongbuk-a Nonchong 한국동북아 논총, vol.XXXVIII, p. 335-337.

JEON Se-young 전세영, 2007, Yulkok-ui Gunjuron 율곡의 군주론 [Le Prince de Yulkok], Séoul, Jipmoondang 집문당.

LEE Hyun-hee 이현희, 1994, Woorinara Kundae Inmulsa 우리나라 근대인물사 [Personnages modernes dans l'histoire de Corée], Séoul, Semunsa 세문사.

LEE Sang-hee 이상희, 1993, «Yulkok-ui Cholhak-gwa Anro» 율곡의 철학과 언로 [«La philosophie et la communication de Yulkok»], Joseon sidae-ui Communication Hyungsang Yeonku 조선시대의 커뮤니케이션 현상연구 [Études de communication de la société Choson], Séoul, Nanam 나남출판사.

LEE Sang-ik 이상익, 2002, "Yulkok-ui Gongronron-gwa Ku Hyundaejeok Uiui» 율곡의 공론론과 그 현대적 의의 [«La théorie de l'opinion publique de Yulkok et son sens moderne »], Yulkok Sasang Yeongu 율곡사상연구 [Étude sur la pensée de Yulkok], vol.V, p. 307-332.

LEE Sang-chul 이상철, 2003, Sinmun-ui Yeoksa 신문의 역사 [L'histoire de la presse], Séoul, Communication Books 커뮤니케이션북스. 
MATSumoto Masao 松本正生, 2003, Yoronchôsa no yukue 世論調査のゆくえ [L'avenir des sondages], Tôkyô, Chûô Kôron 中央公論.

MIYATAKE Michiko 宮武実知子, 2007, «Senkyuhyakuhachijû nendai ikô no yoron kenkyû 1980» 年代以降の「世論」研究 [Recherche sur l'opinion publique depuis les années 1980], p.189-217. Yoron kenkyû to yoronchôsa 輿論研究と世論調査 [L'étude de l'opinion publique et les sondages d'opinion], OKADA Naoyuki 岡田直 之, SATÔ Takumi 佐藤卓己, NISHIHIRA Shigeki 西平重喜 et MIYATAKE Michiko 宮武実 知子, Tôkyô, Shin. yôsha 新曜社.

NISHIHIRA Shigeki 西平重喜, 2009, Yoron wo sagashi motomete 世論をさがし求めて [À la recherche de l'opinion publique], Tôkyô, Minerva shobô ミネルヴャ書房.

OKADA Naoyuki, SATÔ Takumi, NISHIHIRA Shigeki et MIYATAKE Michiko, 2007, Yoron kenkyû to yoron chôsa 輿論研究と世論調査 [L'étude de l'opinion publique et les sondages d'opinion], Tôkyô, Shin. yôsha 新曜社.

SATô Takumi 佐藤卓己, 2007, «Nihon gata yoron no seiritsu»日本型「世論」の成立 [La formation d'une opinion publique de type japonais], p. 85-136, Yoron kenkyû to yoron chôsa 輿論研究と世論調査 [L'étude de l'opinion publique et les sondages d'opinion], OKADA Naoyuki 岡田直之, SATÔ Takumi 佐藤卓己, NISHIHIRA Shigeki 西 平重喜 et MIYATAKE Michiko 宮武実知子, Tôkyô, Shin. yôsha 新曜社.

— 2003, Sengo yoron no media shakaigaku 戦後世論のメディア社会学 [Sociologie des médias de l'opinion publique après la deuxième guerre mondiale], Tôkyô, Kashiwa shobô 柏書房.

SHIBAHARA Takuji, 1975, The Great History of Japan, vol.XXIII, Tôkyô, Shôgakukan 小学 館.

SMITH Anthony, 2005, Segye Sinmun-ui Yeoksa 세계신문의 역사 [The Newspaper: An International History, trad. Choi Jung-ho 최정호], Séoul, Nanam 나남출판사.

SuMITOMO Akifumi 住友陽文, 2001, 近代日本の政治社会の転回 日本史研究 第463号 [«Le renversement politico-social au Japon contemporain »], Étude de l'histoire du Japon, $\mathrm{n}^{\circ} 463$, p. $64-87$.

ҮАмАмото Taketoshi 山本武利, 1992, Shimbun to minshû 新聞と民衆 [La presse et les masses], Tôkyô, Kinokuniya shoten 紀伊国屋書店.

Yi I 이이, 1972, Yulkok Chonsuh 율곡전서, Euvres complètes de Yulkok, Séoul, Publishing Donghwa 동화사.

人物で読む近現代史, Lire l'histoire contemporaine par les personnages, 2001, vol.l, Tôkyô, Aoki shoten青木書店, Société des enseignants d'histoire 歴史教育者協議会.

Le dictionnaire contemporain de la sociologie, Tôkyô, Kôbundô [Koubundou] 弘文堂, 2012 (voir la revue Yoronよろ, nº 90, octobre 2002).

JAPOR (Japan Association for Public Opinion Research), Yoron よろん, nº 90 , octobre 2002.

Dictionnaire chinois-français, 1990, Paris, You-Feng. Gonglun (p. 232), yu. lun (p. 870), min. yi (p. 465).

Dictionnaire des sondages d'opinion (NHK, Nippon Broadcast), 1996, Tôkyô, Ôzorasha 大空社. 
IN-SOOK CHOI

新世纪法汉大词典 Grand dictionnaire français-chinois du nouveau siècle, 1998, Pékin,

Waiyu jiaoxue yu yanjiu chupanshè- 外语教学与研究出版社.

Grand Dictionnaire Japonais 2, 2006, Tôkyô, Shôgakukan 小学館.

Grand Dictionnaire Japonais 3, 2006, Tôkyô, Shôgakukan 小学館.

http://endic.naver.com/krenEntry.nhn?sLn=en\&entryld=802fob58929c424b97abfo

oсзbc871a3\&query=\%EA\%B3\%B $\%$ EB\%A $1 \%$ Ao

http://ci.nii.ac.jp/naid/110006219437, Teidai Shichihakase Jiken 帝大七博士事件.

http://www.kcc.go.kr/user.do : Korea Communications Commission. 